\title{
In-Hospital Telehealth Supports Care for Neonatal Patients in Strict Isolation
}

\author{
Rachel A. Umoren, MBBCh, MS ${ }^{1,2}$ Megan M. Gray, MD ${ }^{1,2}$ Sarah Handley, MD ${ }^{1,2}$ \\ Nathaniel Johnson, RN, NNP-BC ${ }^{1}$ Christina Kunimura ${ }^{1,3}$ Ulrike Mietzsch, MD ${ }^{1,2}$ \\ Zeenia Billimoria, $\mathrm{MD}^{1,2}$ Mark D. Lo, MD ${ }^{1,2}$
}

1 Department of Pediatrics, Seattle Children's Hospital, Seattle, Washington

2 Department of Pediatrics, University of Washington, Seattle, Washington

Address for correspondence Rachel A. Umoren, MBBCh, MS,

3 Digital Health Innovation, Seattle Children's Hospital, Seattle, Washington

Department of Pediatrics, Division of Neonatology, 1959 NE Pacific

Street, Box 356320, Seattle, WA 98195 (e-mail: rumoren@uw.edu).

Am J Perinatol 2020;37:857-860.

\begin{abstract}
Keywords

- telehealth

- pediatrics

- neonatal

- isolation

- COVID-19

The aim of this study is to determine the feasibility of "in-hospital" inpatient telemedicine within a children's referral hospital to facilitate inpatient care activities such as interprofessional rounding and the provision of supportive services such as lactation consultations to pediatric patients in strict isolation. To test the feasibility of in-hospital video telemedicine, a dedicated telemedicine device was set up in the patient's room. This device and the accompanying Bluetooth stethoscope were used by the health care team located just outside the room for inpatient rounding and consultations from supportive services. Video telemedicine facilitated inpatient care and interactions with support services, reducing the number of health care providers with potential exposure to infection and decreasing personal protective equipment use. In the setting of strict isolation for highly infectious viral illness, telemedicine can be used for inpatient care activities such as interprofessional rounding and provision of supportive services.
\end{abstract}

Key Points

- Telehealth supports patient care in isolation.

- Telehealth reduced health care provider exposures.

- Telehealth conserves personal protective equipment.

Video telemedicine has been well described for extending care to pediatric patients in geographically remote settings, including pediatric specialty consults such as neurology, genetics, and neonatology. ${ }^{1-3}$ In these contexts, telemedicine has been demonstrated to be reliable in assessing critically ill children ${ }^{4}$ and increases physician confidence in assessments when compared with telephone consults. ${ }^{5}$

Typical inpatient care activities in the first 24 hours of admission are the admission history and examination, nighttime rounds by the on-call providers to check on the patient's status and morning interprofessional rounds, that is, conducting interdisciplinary discussions, often at the patient's bedside along with parents or caregivers, with physicians, advanced practice providers (APPs), nurses, respiratory therapists, nutrition, pharmacy, physical and occupational therapy and social work to review the patient's course and decide on a plan of care. $^{6}$ The inclusion of learners on daily rounds also increases the overall number of individuals on rounds.

Bedside rounds on patients in isolation are challenging as all the health care providers must wear appropriate personal protective equipment (PPE) including gowns, gloves, masks, and powered air purifying respirators (PAPR) devices, many of which are single use or require extensive cleaning for reuse. No outside communication can occur with providers received

March 28, 2020

accepted

April 1, 2020

published online

April 8, 2020
Copyright $\odot 2020$ by Thieme Medical Publishers, Inc., 333 Seventh Avenue, New York, NY 10001, USA. Tel: +1(212) 760-0888.
DOI https://doi.org/ 10.1055/s-0040-1709687. ISSN $0735-1631$. 
in the isolation room except by phone. Within the room, communication with other health care providers, even those across the room, is difficult due to the fan noise emitted from the PAPR devices. In addition, pediatric and neonatal patient rooms tend to be smaller and less accommodating of larger groups. Delays occur as providers don and doff PPE, and there is an increased exposure risk, particularly risk of contamination during doffing for health care providers, learners, or other team members who may be less familiar with the process. An additional consideration is limiting exposure for other hospitalized patients to reduce the risk of hospital acquired infection. Finally, the need to conserve limited supplies of PPE must be considered. One approach is to limit the number of individuals entering the room to only essential personnel and services such as the attending physician, bedside nurse, and RT (if applicable). In addition, visitors are limited to one parent or caregiver. If the parent or caregiver is ill, they may not visit. Unfortunately, these restrictions decrease access to support services for parents and caregivers and exclude learners from patient care. Remote telerounding in the neonatal intensive care unit (ICU) has been described by Garingo et al, ${ }^{7}$ but there are no known reports on in-hospital telerounding in the same unit. We explored the feasibility of in-hospital telemedicine to provide pediatric care services in the setting of strict isolation within a children's referral hospital.

\section{Materials and Methods}

In February 2020, within the setting of a children's hospital ICU with patients in strict isolation due to concerns for highly infectious viral illness, an InTouch Vici telemedicine cart was used to establish a secure, encrypted connection with a Microsoft Surface Pro tablet device in the ICU. A Littmann 3200 digital bluetooth stethoscope was used with the cart. Consent was obtained from the parent. The patient's nurse was oriented to the use of the stethoscope. The telehealth cart was placed at the bedside ( - Fig. 1). The devices were left in place and were able to be utilized on demand as services were needed. Services working from off-site locations due to quarantine were able to join rounds and visit with the patient's family. After the patient was discharged or removed from isolation, InTouch recommended cleaning protocols were followed for device decontamination using isopropyl alcohol and quaternary ammonium germicidal wipes.

\section{Results}

The interdisciplinary rounding team met outside the room and initiated the video telemedicine connection. The patient was visually examined for alertness, activity, tone, and signs of respiratory distress. With the help of the bedside nurse, the physician auscultated the heart and lung fields. The team discussed the care plan with the input of the bedside nurse who remained in the room. Lactation support and counseling were provided virtually to the mother at the patient's bedside who expressed satisfaction with the service and the information provided. To date, the service has been used for three neonatal ICU patients in strict isolation. This successful trial was instrumental in the deployment of telemedicine to the ICU, emergency department, and special infections unit for patients with suspected or confirmed COVID-19 disease. To meet the demand for additional telehealth devices at a lower cost, Surface Pro (Microsoft) devices equipped with an external microphone/speaker and loaded with InTouch Viewpoint software were deployed to these units.

Given the typical number of providers who might be involved in the care of a critically ill patient over a 24-hour period, the ratio of providers requiring PPE decreased threefold from 21 providers to 7 . This reduction in potential exposures was primarily seen in physician (including resident physicians and medical students), APP, and support services (-Table 1 ).
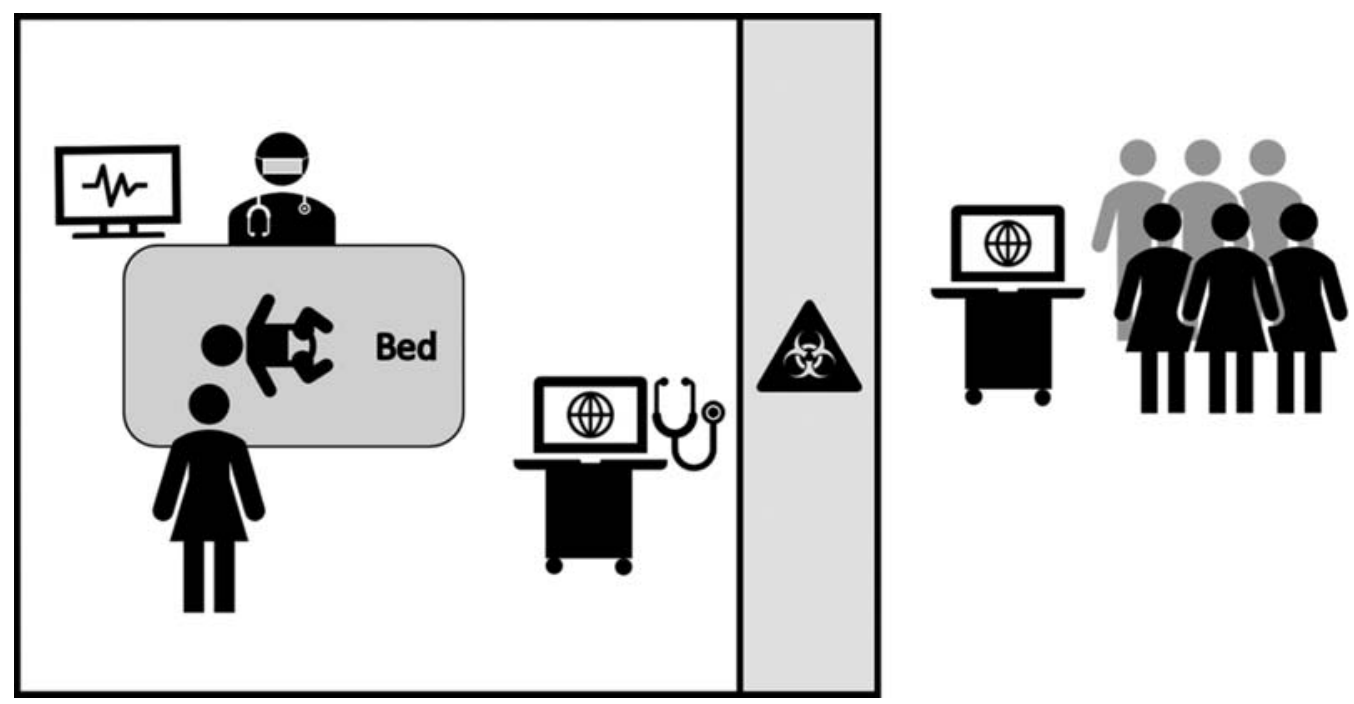

Fig. 1 Telerounding in intensive care unit. 


\begin{tabular}{|c|c|c|}
\hline Type of health care provider & $\begin{array}{l}\text { Without } \\
\text { telehealth }\end{array}$ & $\begin{array}{l}\text { With } \\
\text { telehealth }\end{array}$ \\
\hline Attending physician & 3 & 1 \\
\hline $\begin{array}{l}\text { Fellow/advanced } \\
\text { practice provider }\end{array}$ & 2 & 0 \\
\hline Resident physician & 4 & 0 \\
\hline $\begin{array}{l}\text { Bedside nurse } \\
\text { (admit nurse, charge nurse, } \\
\text { and shift "buddy" nurse) }\end{array}$ & 5 & 4 \\
\hline Respiratory therapist & 2 & 2 \\
\hline $\begin{array}{l}\text { Support services } \\
\text { (lactation, pharmacy, } \\
\text { occupational/physical therapy, } \\
\text { nutrition, and social work) }\end{array}$ & 5 & 0 \\
\hline Total & 21 & 7 \\
\hline
\end{tabular}

\section{Discussion}

The need to provide family-centered care and maintain supportive services for pediatric patients in response to COVID-19 led us to successfully pilot inpatient telemedicine for neonatal patients in strict isolation. We found that the number of health care providers, who needed to utilize PPE in the care of the patient in isolation, decreased substantially with the use of telehealth. These findings are likely reflective of the number of health care providers, learners, and supportive staff involved in patient care at a large academic center. While nonacademic facilities may have fewer individuals involved in care, they also tend to have fewer health care providers available to serve in back-up roles, increasing the risk of potential exposures and health care provider illness. Community hospitals may also be less equipped to care for patients in isolation. ${ }^{8,9}$ This means that reducing exposure risk and conserving PPE is even more critical for smaller health care facilities.

Telemedicine can also be used to ensure continued access to inpatient supportive services such as nutrition, occupational and physical therapy and social work for families of patients in strict isolation. While these consulting services may provide direct input to bedside rounds, they are also frequently involved in direct care and counseling for parents. When access to these services are limited due to the need for "social distancing" or to decrease exposure risk, both for the patient in isolation and for other patients in the hospital who receive these services, this may impact patient care. In the ICU setting, having fewer providers "tied up" following the strict isolation recommendations, particularly donning and doffing, frees up some providers to quickly assist with other emergencies in the ICU adding to patient safety of nonexposed patients as well. In addition, some support service team members were able to work from quarantine using telehealth and remain engaged in patient care decreasing the risk of staff shortages.
Using telemedicine, health care providers and support services were able to converse with the family directly without masks and other PPE, obscuring their faces or limiting audibility. In our pilot, the patient's family had positive impressions of the use of telemedicine. Patients and families have demonstrated positive responses toward telemedicine in prior studies. $^{10,11}$ Video calls or livestreaming video of premature newborns has also been described for family support during long NICU hospitalizations. ${ }^{12}$ With many health care facilities limiting in-patient visitation in response to the COVID-19 pandemic, telemedicine could be used to support families who cannot visit due to exposure concerns. Formal evaluations of family satisfaction with inpatient services provided via telemedicine, and the degree to which this supports patient and family-centered care are needed. Additional studies on methods to provide the full scope of inpatient services via telemedicine are important to the rapid roll out of this technology during the COVID-19 pandemic.

\section{Conclusion}

Inpatient telemedicine in the setting of strict isolation for highly infectious viral illnesses is feasible, reduces the use of PPE, and minimizes exposure of essential health care providers, while still providing comprehensive family centered care to affected patients. More studies are needed to explore this novel pediatric use of in-patient telemedicine.

\section{Authors' Contributions}

R.A.U. had full access to all the data in the study and takes responsibility for the integrity of the data and the accuracy of the data analysis. C.K. provided administrative, technical, or material support. R.A.U. dedicated in statistical analysis and carried out study supervision. All authors have made substantial contributions to study concept and design; acquisition, analysis, or interpretation of data; drafting of the manuscript; and critical revision of the manuscript for important intellectual content.

\section{Conflict of Interest}

None declared.

\section{Acknowledgments}

The authors would like to acknowledge the Seattle Children's Information Technology and Telehealth teams for their technical and administrative support.

\section{References}

1 Bell RC, Yager PH, Clark ME, et al. Telemedicine versus face-to-face evaluations by respiratory therapists of mechanically ventilated neonates and children: a pilot study.(Report). Respir Care 2016;61 (02):149-154

2 Wenger TL, Gerdes J, Taub K, Swarr DT, Deardorff MA, Abend NS. Telemedicine for genetic and neurologic evaluation in the neonatal intensive care unit. J Perinatol 2014;34(03):234-240

3 Fang JL, Collura CA, Johnson RV, et al. Emergency video telemedicine consultation for newborn resuscitations: the Mayo Clinic experience. Mayo Clin Proc 2016;91(12):1735-1743 
4 Siew L, Hsiao A, McCarthy P, Agarwal A, Lee E, Chen L. Reliability of telemedicine in the assessment of seriously ill children. Pediatrics 2016;137(3):e20150712

5 Umoren RA, Gray MM, Schooley N, Billimoria Z, Smith KM, Sawyer TL. Effect of video-based telemedicine on transport management of simulated newborns. Air Med J 2018;37(05):317-320

6 Voos KC, Ross G, Ward MJ, Yohay A-L, Osorio SN, Perlman JM. Effects of implementing family-centered rounds (FCRs) in a neonatal intensive care unit (NICU). J Matern Fetal Neonatal Med 2011;24(11):1403-1406

7 Garingo A, Friedlich P, Chavez T, et al. "Tele-rounding" with a remotely controlled mobile robot in the neonatal intensive care unit. J Telemed Telecare 2016;22(02):132-138

8 Katznelson JH, Wang J, Stevens MW, Mills WA. Improving pediatric preparedness in critical access hospital emergency depart- ments: impact of a longitudinal in situ simulation program. Pediatr Emerg Care 2018;34(01):17-20

9 Obaid JM, Bailey G, Wheeler $\mathrm{H}$, et al. Utilization of functional exercises to build regional emergency preparedness among rural health organizations in the US. Prehosp Disaster Med 2017;32(02):224-230

10 Whitten P, Love B. Patient and provider satisfaction with the use of telemedicine: overview and rationale for cautious enthusiasm. J Postgrad Med 2005;51(04):294-300

11 Varkey P, Schumacher K, Swanton C, Timm B, Hagen PT. Telemedicine in the work site: a study of feasibility, and patient and provider satisfaction. J Telemed Telecare 2008;14(06):322-325

12 Epstein EG, Arechiga J, Dancy M, Simon J, Wilson D, Alhusen JL. Integrative review of technology to support communication with parents of infants in the NICU. J Obstet Gynecol Neonatal Nurs 2017;46(03):357-366 